Vol. 11

$54-60$

Enero - Diciembre de 2015

\title{
ESTRATEGIA DIDÁCTICA PARA LA ENSEÑANZAY APRENDIZAJE EN EL LABORATORIO DE CIRCUITOS ELECTRICOS DE LA UNIVERSIDAD DE LA COSTA CUC
}

\author{
TEACHING STRATEGY FOR LEARNING IN THE LABORATORY ELECTRICAL CIRCUITS \\ UNIVERSIDAD DE LA COSTA CUC
}

\author{
Rita Dederlé-Caballero*, Ebaldo Pérez-Villareal ${ }^{* *}$, Sandra Lora-Castro***, \\ Carlos Peña-Arrieta ${ }^{\star * * *}$ y Fernando Charris-Chiquillo ${ }^{\star * * * *}$
}

\begin{abstract}
RESUMEN
Este artículo es producto de una investigación que pretende brindar nuevos elementos didácticos a través de un módulo orientador basados en las TIC para laboratorio de circuitos eléctricos del Programa de Ingeniería Eléctrica de la Universidad de la Costa CUC.

La investigación inicia identificando las estrategias que actualmente se están impartiendo en los laboratorios en el marco de las investigaciones realizadas a nivel regional, nacional e internacional, y se desarrolla un método de investigación descriptivo cuantitativo. La investigación demostró la necesidad de proponer un módulo orientador que les permita a los estudiantes construir su propio conocimiento, de esta manera se invita al estudiante a ser autónomo, que investigue, reflexione y sea crítico de su práctica, el papel del docente es de facilitador apoyado con los módulos propuestos en esta investigación.

De acuerdo a los resultados arrojados, se evidencia que se requiere promover y difundir en la asignatura de laboratorio de circuitos eléctricos el apoyo de las TIC para el logro de aprendizajes significativos, el cual da un vuelco a la metodología tradicional de enseñanza, y de acuerdo a esto vemos que el uso de estas herramientas permite garantizar un mejoramiento en el rendimiento académico.
\end{abstract}

Palabras clave: Estrategia didáctica, enseñanza y aprendizaje, módulo orientador laboratorio de circuitos eléctricos, TIC.

Fecha de recepción: Abril 10 de 2015 / Fecha de aceptación: Mayo 22 de 2015

Tipología: Artículo de Investigación Científica y Tecnológica

Para citar este artículo: Dederlé, C. R., Pérez, V. E., Lora, C. S., Peña, A. C., \& Charris, Ch. F. (2015). Estrategia didáctica para la enseñanza y aprendizaje en el laboratorio de circuitos eléctricos de la Universidad de la costa CUC. Praxis. Vol. 11, 54 - 60

\footnotetext{
*Mg. En Educación, Departamento de Ciencias Básicas, Grupo de investigación PRODUCOM Universidad de la costa CUC, Barranquilla, Colombia. Email: ritadederle@gmail.com

**Mg. En Educación, Departamento de Ciencias Básicas, Grupo de investigación PRODUCOM Universidad de la costa CUC, Barranquilla, Colombia. Email: ebald025@hotmail.com

***Mg. En Informática Educativa, Departamento de Ciencias Básicas, Grupo de investigación PRODUCOM, Universidad de la costa CUC, Barranquilla, Colombia.Email: sallyluz@gmail.com

****Mg. En Informática Educativa, Departamento de Ciencias Básicas, Grupo de investigación PRODUCOM Universidad de la costa CUC, Barranquilla, Colombia. Email:carlospenaarrieta@gmail.com

*****Esp. En estudios Pedagógicos Departamento de Ciencias Básicas, Grupo de investigación PRODUCOM Universidad de la costa CUC, Barranquilla, Colombia.Email: fernandocharris73@gmail.com
} 


\begin{abstract}
This paper is the result of a research that aim new didactical elements through a guiding module using TIC (Technologies of Information and Communication) for the electrical circuit laboratories of the faculty of Electrical Engineering in the Universidad de la Costa.

The research begins identifying the current laboratories strategies in the local, national and international context using a descriptive-quantitative research methodology. This work showed the need to propose a guiding module that help students to develop the own knowledge, even helps to be autonomous to investigate, meditate and criticize the practice, and the role of the teacher is give a support with the proposals module guides in this research.

According to the results, it is required a promotion and diffusion of the electrical circuits course using TIC to get the goal of a significant learning, that transform the traditional teaching methodology with this it is noted that using this tools ensure an improvement in the academicals performance.
\end{abstract}

Keywords: teaching strategy, teaching and learning, guiding electrical circuits lab module, TIC.

\section{INTRODUCCIÓN}

$\mathrm{L}$ a enseñanza y aprendizaje de asignaturas prácticas relacionadas con la física, en especial las de laboratorio de circuitos eléctricos, son temas que preocupan y dan pie para investigar los factores que inciden en el proceso de enseñanza-aprendizaje durante la realización de estos laboratorios, con la finalidad de proponer estrategias didácticas que favorezcan el rendimiento académico de los estudiantes y al mismo tiempo facilite la labor del docente enriqueciendo su acción pedagógica a través de elementos y recursos proporcionados por las TIC, además de empalmar las estrategias propuestas con el modelo pedagógico desarrollista, de la Universidad de la Costa CUC.

Las propuestas didácticas bajo el modelo pedagógico, acorde con los planteamientos expuestos por este modelo, busca fortalecer la autonomía de los estudiantes para que puedan desarrollarse en las estructuras cognitivas a través de la experiencia individual, donde el alumno es el centro del proceso y constructor de su propio conocimiento, además se incentiva el trabajo colaborativo, lo cual posibilita el desarrollo del conocimiento a través de saberes teóricos y prácticos, permitiendo mejorar la relación y comunicación.
De acuerdo a lo anterior es importante resaltar también la labor del docente, donde su rol es de guía facilita el desarrollo de la estructura del pensamiento de sus estudiantes.

El docente perfilado de esta manera implica que en cada acto educativo se deben analizar las condiciones del grupo, del contexto donde se desenvuelve para diseñar cada uno de estos actos y además se requiere que sea innovador con un espíritu investigativo, de esta manera se crea la necesidad de fomentar el sentido ético y la responsabilidad que tiene un docente con sus estudiantes.

En este sentido para apoyar la intencionalidad se proponen estrategias didácticas para el proceso de enseñanza y aprendizaje en los laboratorios de circuitos eléctricos del Programa de Ingeniería Eléctrica, debemos tener en cuenta tres aspectos claves que son: la didáctica de los laboratorios eléctricos, la aplicación de las TIC y las teorías de aprendizaje que sustenta el modelo desarrollista de la Universidad de la Costa CUC.

En primera instancia es indudable la participación de las TIC en diversos campos de nuestra sociedad, esto conlleva a la necesidad de fortalecer las políticas de incorporación del uso de las tecnologías en nuestro sistema educativo. 
De acuerdo a lo anterior, la universidad ha hecho inversiones de implementación en recursos tecnológicos para apoyar el proceso de enseñanza-aprendizaje, como es el caso de la plataforma moodle, donde las TIC han sido un apoyo para la orientación de estos conceptos. Sin embargo, valorando el uso de las TIC como herramienta facilitadora en el proceso de enseñanza y aprendizaje se hace indispensable que forme parte de nuestra propuesta pedagógica.

\section{Didácticas de los laboratorios de circuitos eléctricos y lineamientos Curriculares}

Dada la naturaleza experimental de la asignatura laboratorio de circuitos eléctricos, es de esperar que la didáctica adecuada esté basada en el amplio manejo de los dispositivos y recursos con que cuenta la institución, enmarcado dentro del modelo pedagógico desarrollista, privilegiando la iniciativa y el ingenio de los estudiantes para que realicen las prácticas a manera de solución de problemas.

Es importante resaltar el papel fundamental que juegan los medios didácticos, los cuales constituyen la serie de recursos utilizados para favorecer el proceso, de acuerdo a esto su utilización depende del objetivo trazado en la planeación curricular y la intencionalidad didáctica.

En este sentido no es desconocida la existencia de una gran variedad de matices didácticas, puestas a disposición del docente, quien juega un papel importante, ya que al tener a su disposición la amplia gama de posibilidades, su compromiso es propiciar la mejor manera de aplicar cada una de ellas, teniendo claro los objetivos propuestos y las competencias que busca fortalecer en el estudiante.

En este sentido las estrategias didácticas planteadas por el docente, apoyado con la gama de recursos, deben apuntar a producir un aprendizaje significativo.

La clave de la acción pedagógica depende en gran medida de la participación que se logre del alumnado, y de la motivación e interés trasmitido por el docente. Sin embargo, el estudiante está sometido a una presión intensa, de modo que su objetivo final es cuantitativo, obtener una valoración cuantitativa para aprobar. En vista de esta situación es importante gestionar la motivación que los lleve a interesarse por aprender y concientizarlos que los espera un mercado competitivo.

De acuerdo a lo anterior el laboratorio es un escenario para poner en práctica situaciones reales y de ahí la importancia de recrear situaciones que lo inviten a la reflexión y análisis.

Para Hodson (1994) el trabajo práctico de laboratorio sirve:

- Para motivar, mediante la estimulación del interés y la diversión

- Para enseñar las técnicas de laboratorio

- Para intensificar el aprendizaje de los conocimientos científicos

- Para proporcionar una idea sobre el método científico, y desarrollar la habilidad en su utilización

- Para desarrollar determinadas "actitudes científicas”, tales como la consideración de las ideas y sugerencias de otras personas, la objetividad y la buena disposición para no emitir juicios apresurados (p.300)

\section{Estrategias virtuales y uso de las TIC en el laboratorio de circuitos eléctricos}

Existen muchas aplicaciones específicas para la simulación de circuitos eléctricos que se pueden emplear de forma complementaria para hacer el análisis de los circuitos eléctricos. Usando la plataforma moodle como vía de comunicación, mediante los enlaces se puede orientar a los estudiantes en el uso de las TIC para la realización de las prácticas virtualmente, para luego verificar con los montajes físicos y viceversa.

\section{Las nuevas tecnologías y la educación}

La tecnología como fenómeno cultural, es el resultado de aplicar un conjunto de conocimientos en la creación de instrumentos que ha hecho posible la transformación de la naturaleza 
por el hombre, y que son susceptibles de ser estudiados, comprendidos y mejorados por las generaciones presentes y futuras (MEN, 1999, p. 25).

La educación en tecnología se presenta como un proceso innovador dentro de las reformas educativas, cuyo objetivo es desarrollar un modelo de pensamiento que busque relacionar los conocimientos adquiridos del hombre con el hacer, permitiéndole de esta manera el logro de competencias.

La incorporación de las TIC en la educación permite adquirir conocimientos a través de sus recursos, esto implica una escuela abierta a transformaciones con procesos flexibles presentando estrategias que induzcan a mejorar el proceso de enseñanza-aprendizaje, donde se hagan presentes los valores y se satisfagan las necesidades de cada individuo.

El uso de herramientas en la educación se ha extendido y por ello es cada vez más frecuente encontrar en instituciones educativas ordenadores actualizados, pizarras digitales, software aplicativos etc., los cuales han iniciado transformaciones en el proceso de enseñanzaaprendizaje, esto es un cambio en la forma de generar y difundir conocimientos.

Debido a la inmersión de las tecnologías en la educación surge el reto de conocer, entender e implementar la forma adecuada en que éstos medios tecnológicos (computadoras personales, video proyectores, plataformas en línea, la web; entre otros) nos llevan a dar soporte a las actividades de enseñanza-aprendizaje que buscan el desarrollo de capacidades y habilidades en los alumnos de educación superior (Islas, 2008, p.1).

\section{Propósitos de la educación en tecnología}

En la actualidad estamos inmersos en un mundo llamado la "sociedad de la información", el asombroso desarrollo de las nuevas tecnologías implican una transformación en la sociedad y esto conlleva a grandes cambios en los diferentes ámbitos de la vida humana, entre estos la comunicación, la manera de encontrar información, la industria, el comercio y ante todo la educación.

En este sentido se puede considerar el propósito de la educación en tecnología como una reflexión desde la educación sobre el impacto que ha generado ante la sociedad el desarrollo de la tecnología, y lo imprescindible que es educar al hombre para ser competente en esta sociedad. Se propone además el logro de una cultura en las TIC, lo que implica que las instituciones asuman la responsabilidad de este reto e incorporen en su currículo el uso de las nuevas herramientas tecnológicas, el cual debe ser parte de los objetivos que se ha añadido dentro del plan de mejoramiento de muchas instituciones la inclusión de las TIC para fortalecer el proceso de enseñanza y aprendizaje.

\section{Teoría del aprendizaje: aprendizaje significativo}

La teoría del aprendizaje significativo brinda herramientas que conducen a una mejor aprehensión del conocimiento, está basado en la importancia del rol que desempeña el estudiante como principal protagonista de su conocimiento y el papel orientador del docente.

Su participación en esta propuesta es al apoyo que brinda en la asignatura del laboratorio de física de circuitos eléctricos, las cuales permiten propiciar los niveles de conocimiento.

Un aprendizaje es significativo cuando los contenidos son relacionados de modo no arbitrario y sustancial con lo que el alumno ya conoce. Por relación sustancial se debe entender que las ideas se relacionan con algún aspecto existente específicamente relevante de la estructura cognoscitiva del alumno, como una imagen, un símbolo ya significativo, un concepto o una proposición (Ausubel-Novak, 1983). Esto quiere decir que en el proceso educativo, es importante considerar lo que el individuo ya sabe de tal manera que establezca una relación con aquello que debe aprender. Este proceso tiene lugar si el educando tiene en su estructura cognitiva conceptos, estos son: ideas, proposiciones, estables y definidos, con 
los cuales la nueva información puede interactuar y desarrollar con todo esto la creatividad y por consecuencia la innovación (Merchan, 2011, p.4).

Por esta razón se requiere que los jóvenes no sólo aprendan una cantidad de conocimientos científicos y matemáticos tradicionales, sino que puedan resolver situaciones que conduzcan al uso adecuado de esos conocimientos y habilidades científicas y matemáticas para desenvolverse en la vida cotidiana y profesional; lo que lleva a la búsqueda de factores o aspectos relevantes que hagan del trabajo del aula y fuera de ella una cotidianidad, un aprender de verdad y aún más, un placer académico, lo que conduce a atender, especialmente, los estilos de aprendizajes.

\section{El constructivismo como una estrategia metodológica que posibilita el aprendizaje significativo}

Entendida la estrategia metodológica como el procedimiento material que se aborda para aproximarse a un nivel de coherencias entre teoría y práctica, el constructivismo supone que el conocimiento está en la mente, en el interior del sujeto y que sus ideas previas son un punto de partida, y lo que hay que posibilitar es el desarrollo de sus potencialidades cognitivas, afectivas, psicomotrices y volitivas.

La concepción constructivista se organiza en torno a tres ideas fundamentales:

1. El alumno es el responsable último de su propio proceso de aprendizaje. Él es quien construye (o más bien reconstruye) los saberes de su grupo cultural, y éste puede ser un sujeto activo cuando manipula, explora, descubre o inventa, incluso cuando lee o escucha la exposición de los otros.

2. La actividad mental constructiva del alumno se aplica a contenidos que poseen ya un grado considerable de elaboración. Esto quiere decir que el alumno no tiene en todo momento que descubrir o inventar en un sentido literal todo el conocimiento escolar.

3. La función del docente es engarzar los procesos de construcción del alumno con el saber colectivo culturalmente organizado. Esto implica que la función del profesor no se limita a crear condiciones óptimas para que el alumno despliegue una actividad mental constructiva, sino que debe orientar y guiar explícita y deliberadamente dicha actividad. (Díaz, 2011, p.5)

\section{METODOLOGÍA}

De acuerdo al enfoque presentado en el marco teórico, se resalta la importancia de que el profesor considere a su estudiante el protagonista de su propio aprendizaje, y para ello debe utilizar estrategias y recursos adecuados apoyados en las TIC para lograr que sus estudiantes sean sujetos activos en este proceso de enseñanza-aprendizaje.

En este sentido la investigación busca proponer estrategias didácticas para el proceso de enseñanza y aprendizaje en los laboratorios de circuitos eléctricos en el programa de ingeniería eléctrica de la Universidad de la Costa CUC, en la ciudad de Barranquilla, en Colombia. Por lo tanto la metodología del trabajo de la presente investigación es de tipo cuantitativo, considerando que los fenómenos a estudiar son cuantificables debido a que el procedimiento conlleva a la medición de ciertas variables a través de la determinación de indicadores específicos, la forma objetiva de tomar los datos y llegar a las conclusiones.

Además de lo anterior, la presente investigación se basa en las dificultades que presentan los estudiantes en apropiarse de conceptos físicos, los cuales, como se mencionó anteriormente, son de gran abstracción.

La investigación adquiere relevancia en la medida en que van surgiendo en su desarrollo aspectos importantes y estos analizados desde una perspectiva cuantitativa, lo que nos permite conocer los componentes presentes en el problema de investigación.

De acuerdo a esto el termino investigación que, en general, significa indagar o buscar, cuando se aplica a las ciencias sociales, toma la connotación específica de crear conocimientos sobre 
la realidad social, es decir, sobre su estructura, las relaciones entre sus componentes, su funcionamiento, los cambios que experimenta el sistema en su totalidad o en esos componentes. Briones (1996, p. 17)

La investigación se presenta bajo un paradigma cuantitativo. El método de investigación es descriptivo de campo donde se apunta a explorar y describir la importancia de proponer estrategias didácticas apoyadas en las TIC, las cuales se sugiere sean utilizadas como herramientas pedagógicas en la asignatura de laboratorios de circuitos eléctricos en el programa de Ingeniería eléctrica de la Universidad de la Costa CUC, en la ciudad de Barranquilla, en Colombia. El diseño es no experimental, debido a que se trata de una investigación cuantitativa, donde no hay manipulación deliberada de variables, y donde se observa el fenómeno tal y como se muestra en su contexto natural para posteriormente analizarlo, se consideró la elaboración de una encuesta.

La población corresponde al total de los estudiantes de tercer semestre de Ingeniería eléctrica de la jornada diurna que cursan la asignatura de circuitos eléctricos de la Universidad de la Costa. CUC en el 2014-1, con una población de 40 estudiantes de ambos sexos cuyas edades están entre los 18 y 23, para la investigación se toma todos los 40 estudiantes.

Después de las encuestas a los estudiantes, se procede a tabular los datos para hacer un análisis de los mismos, se utilizó el Excel en la realización de tablas y gráficas, y posteriormente analizar los resultados de los mismos, para luego sacar las conclusiones.

\section{RESULTADOS}

En coherencia con lo propuesto en la investigación se formularon preguntas direccionadoras que nos invitan a reflexionar acerca de la metodología que actualmente se está impartiendo en la asignatura de laboratorios de circuitos eléctricos, el cual presentó un alto porcentaje en la metodología de tipo magistral, por otro lado los estudiantes manifestaron otros aspectos a mejorar como la motivación, incentivar el trabajo colaborativo, la actualización del docente en el manejo de las TIC como apoyo al proceso didáctico a través del uso de la plataforma moodle, propiciar espacios de participación y discusión de los resultados a través de preguntas enmarcadas en contextos reales, la importancia de socializar y valorar el uso de los pre-informes para verificar los conocimientos previos de los estudiantes, además de analizar y debatir los resultados del informe final.

\section{CONCLUSIONES Y SUGERENCIAS}

Evidenciamos que se requiere promover y difundir en la asignatura de laboratorios de circuitos eléctricos el apoyo de las TIC para el logro de aprendizajes significativos, el cual da un vuelco a la metodología tradicional de enseñanza, y de acuerdo a esto se considera que el uso de estas herramientas permitan garantizar un mejoramiento en el rendimiento académico.

De igual forma, las TIC atienden las diferentes formas de aprender de los estudiantes, de acuerdo a sus niveles de inteligencia, particularidades y necesidades, sintiéndose atraídos por la forma cómo estas herramientas les brindan diferentes opciones, como: representaciones visuales de gráficas, imágenes, animaciones, simulaciones; proporcionando alto grado de realidad psicológica, propiciando a la mente alcanzar los objetivos de una forma agradable y sencilla.

Estas herramientas permiten al estudiante "aprender a aprender", entrando al mundo de internet donde puede encontrar archivos, videos tutoriales del tema estudiado donde aprende descubriendo, al estimular la independencia y el auto-aprendizaje.

Sin embargo es de anotar que aunque las TIC inciden positivamente en el logro del aprendizaje de los jóvenes, es menester recalcar la importancia de la mediación del docente como elemento orientador. Por lo tanto, es indispensable que el docente sea competente para las exigencias que acarrea esta nueva era, como tener claro conceptualizaciones propias de la asignatura y la forma de aplicar estos conceptos, emplear estrategias específicas para el uso efectivo de 
las TIC, y fomentar una cultura de utilización de herramientas TIC.

Esta investigación sirve como evidencia para proponer los "Módulos orientadores del proceso de enseñanza aprendizaje de los laboratorios de circuitos eléctricos" y mejorar el manejo de algunas actividades de la plataforma virtual Moodle en especial las actividades evaluativas.

De igual manera resaltar la importancia de la capacitación en los docentes en especial el manejo de actividades multimedia para enriquecer el trabajo en el aula fortaleciendo aún más la motivación y el interés por parte de los estudiantes.

Por su parte es importante que el docente atienda las características individuales de cada estudiante y hacer un diagnóstico a sus necesidades.

Es importante que el docente evalúe continuamente su proceso pedagógico, prepare sus clases de una manera organizada donde incluya situaciones problemas mediadas con estrategias didácticas apoyadas en TIC.

Son diversas las herramientas que sirven de apoyo al docente, pero es indispensable identificar el tipo de herramienta que sea pertinente de acuerdo al tema tratado.

La motivación juega un papel importante, por lo tanto se sugiere escoger en la acción pedagógica las herramientas adecuadas que fomente la participación de los estudiantes.

Se sugiere además hacer uso de la creatividad, ser innovadores y tener un espíritu investigativo, esto es experimentar en clases en búsqueda de nuevas estrategias didácticas y nuevas posibilidades de utilización de recursos TIC.

Se propone un módulo orientador que contenga todas las guías de laboratorio que se deben realizar en las respectivas asignaturas. Este documento es avalado por la Directora de Programa de Ingeniería Eléctrica y por los docentes que orientan las asignaturas de laboratorio. Las prácticas que se proponen tratan de inducir al estudiante a que redescubra los principios, leyes o teoremas empleados en la solución y análisis de circuitos.

\section{REFERENCIAS BIBLIOGRAFICAS}

Ausbel, N. (1983). Psicología Educativa. Un punto de vista cognoscitivo $2^{\circ}$. México: Trillas

Díaz, F. (2011). Constructivismo y Aprendizaje significativo Artículo de revista cmap, Recuperado el 18 junio de 2015, de http://cmapspublic2.ihmc.us/ rid $=1249740839640 \_870475537 \_5794 /$ constructivismoyaprendizajesignificativo.pdf

Briones, G. (1996). Métodos y Técnicas de Investigación para Ciencias Sociales. Recuperado el 2 de junio de 2014, de: http://www.textosdigitales.com.ar/CP/ CICLO_BASICO/3.017___Fundamentos_Tecnicos/ Briones_-_Metodos_y_Tecnicas_de_Investigacion. pdf

Hodson, D. (1994). Investigación y Experiencias Didácticas. Hacia un enfoque crítico del trabajo de laboratorio. Recuperado el 25 de mayo de 2014, de: http://www.raco.cat/index.php/ensenanza/ article/viewFile/21370/93326

Islas, C. (2008) . ¿Garantía de eficacia en los procesos de enseñanza aprendizaje en la educación superior? Artículo de revista Uso de las tecnologías (en la educación) Recuperado el 18 junio de 2015, de http://www.eveliux.com/mx/Uso-deTecnologias-en-la-educacion.html

Merchán, E. (2011). Aprendizaje significativo apoyado en la creatividad e innovación Artículo de revista Metodología de la ciencia, Recuperado el 18 junio de 2015, de http://www.ammci.org.mx/ revista/pdf/Numero3/4art.pdf

Ministerio de Educación Nacional. Una propuesta para una educación sobre tecnología, ciencia y técnica. Santafé de Bogotá, 1999, de http:// www.mineducacion.gov.co/proyectos/1737/articles-2767_recurso_nuevo_14.pdf 